\begin{tabular}{|c|l|}
\hline Title & An oligochaete homologue of the Brachyury gene is expressed transiently in the third quartette of micromeres \\
\hline Author(s) & Kitakoshi, Tomohito; Shimizu, Takashi \\
\hline Citation & $\begin{array}{l}\text { Gene Expression Patterns, 10(6), 306-313 } \\
\text { https://doi.org/10.1016j.gep.2010.07.001 }\end{array}$ \\
\hline Issue Date & 2010-09 \\
\hline Doc URL & http://hdl.handle.net/2115/43800 \\
\hline Type & article (author version) \\
\hline File Information & GEP10-6_306-313.pdf \\
\hline
\end{tabular}

Instructions for use 


\title{
An oligochaete homologue of the Brachyury gene is expressed transiently in the third quartette of micromeres
}

\author{
by \\ Tomohito Kitakoshi, Takashi Shimizu* \\ Division of Biological Sciences, Graduate School of Science, Hokkaido University, \\ Kita-10, Nishi-8, Kita-ku, Sapporo 060-0810, Japan
}

*Corresponding athor. Tel.: +81-11-706-4460. Fax: +81-11-706-4851.

E-mail address: stak@sci.hokudai.ac.jp (T. Shimizu). 


\begin{abstract}
We have isolated a Brachyury homologue (Ttu-Bra) from the oligochaete annelid Tubifex tubifex which displays a direct mode of development. Developmental RT-PCR analysis showed that Ttu-Bra transcripts are present in embryos at stages 9-11 and 16-17, but undetectable at the remaining embryonic stages. Whole-mount in situ hybridization demonstrated that Ttu-Bra is expressed transiently in the third quartette of micromeres, which are located at the prospective stomodaeum (at stages 9-11). The second burst of Ttu-Bra expression occurs at the posterior end of stage 16 embryo that undergoes body elongation. Ttu-Bra-expressing cells, which are organized in a circle at stage 16, become aggregated at the proctodaeum at stage 17. Consistent with the results of the RT-PCR analysis, there is no sign of Ttu-Bra-expressing cells in embryos that undergo gastrulation during stages 12 to 15.
\end{abstract}

Keywords:

Brachyury, Annelid, Tubifex tubifex, Micromeres, Teloblasts, Blastopore, Direct development

\title{
1. Results and Discussion
}

The gene Brachyury encodes a transcriptional factor that has a conserved DNA binding domain called T-domain in N-terminal half of the protein. Although Brachyury homologues have been isolated in many metazoan phyla, their developmental roles have been analyzed only in Drosophila and vertebrates. In Drosophila, brachyenteron (Brachyury homologue) is involved in morphogenesis of the caudal part of the gut and its derivatives (e.g. anal pads) and the development of the caudal visceral mesoderm (Kispert et al., 1994; Kusch 
and Reuter, 1999). In vertebrates, Brachyury has a conserved role in mesoderm differentiation (Showell et al., 2004).

The spatial patterns of Brachyury expression have been examined in many organisms. Most intriguingly, in all of the animals examined, Brachyury is expressed around the blastopore of the developing embryos and larvae. Recent comparative analyses of Brachyury expression patterns in basal members of Lophotrochozoa, Ecdysozoa and Deuterostomia, have suggested that the original expression of Brachyury in Bilaterians was in the blastopore and its derived structures, the stomodaeum/foregut and the proctodaeum/hindgut in the primary larva (Arendt et al., 2001; Technau, 2001; Scholz and Technau, 2003). Such “original” traits of Brachyury expression appear to have been preserved in animals that display an indirect mode of development via primary, ciliary larvae (such as trochophora, bipinnaria, pluteus, and tornaria larvae); these animals include the polychaete annelid Platynereis dumerilli (Arendt et al., 2001), the molluscs Patella vulgata (Lartillot et al., 2002) and Saccostrea kegaki (Kin et al., 2009), the hemichordate Ptychodera flava (Tagawa et al., 1998), the starfish Asterina pectinifera (Shoguchi et al., 1999), and the sea urchins Lytechinus variegatus (Gross and McClay, 2001) and Paracentrotus lividus (Croce et al., 2001). In the acoel flatworm Convolutriloba longifissura which has only one opening to its digestive system, Brachyury homologue is expressed not only in association with the mouth but also in small region at the posterior end of the animal (Hejnol and Martindale, 2008). In contrast, in animals that display a direct mode of development without larval stages or develop indirectly by means of larvae other than ciliary larvae (such as tunicates, acraniates, vertebrates, insects and nematodes), there is no stomodeal Brachyury 
expression; Brachyury expression is restricted to the small caudal region (Yasuo and Satoh, 1994; Holland et al., 1995; Kispert et al., 1994; Woollard and Hodgkin, 2000). It should be mentioned, however, that there has been reported a directly developing organism (a chaetognath Paraspadella gotoi) in which a Brachyury homologue is expressed not only in the blastopore region but also in the stomodaeum region (Takada et al., 2002).

During the course of examining temporal and spatial aspects of embryonic expression of a Brachyury homologue (Ttu-Bra) in an oligochaete annelid Tubifex tubifex which uses a direct mode of development via teloblasts, we found that Ttu-Bra is expressed not only in a small ring of cells encircling the caudal region but also in an anterior region corresponding to the prospective stomodaeum; this expression pattern is apparently similar to the expression patterns reported for organisms displaying the indirect mode of development through ciliary larvae.

\subsection{Cloning of Tubifex homologue of Brachyury}

Using a set of degenerate oligonucleotide primers, we amplified a Brachyury homologue from T. tubifex cDNA generated from mixed embryonic stages. The amplified fragment was $361 \mathrm{bp}$ long and the deduced amino acid sequence contained a putative T-domain, which was more homologous to T-domain of Brachyury than that of other T-box family members.

To obtain additional sequence for the entire T-domain, not only 3'RACE but also 5’RACE were performed using gene-specific primers. The amplified fragments contained 1419 bp in 3'RACE and 661 bp in 5'RACE. Nucleotide sequences thus obtained were 
aligned with each other using an AutoAssembler 2.0 software (Applied Biosystems). The results showed that a cDNA of T. tubifex homologue of Brachyury (Ttu-Bra) contained 2100 bp (except for a poly-A tail) and that an open reading frame of 1524 bp encodes a polypeptide of 508 amino acids (Accession No.AB553740).

The T-domain of Ttu-Bra aligned well with Brachyury proteins of other animals (such as molluscs, polychaete annelids, insects and vertebrates) with more than 69\% identity (Fig. 1A), suggesting that Ttu-Bra is a true Brachyury homologue. Supporting this notion, Ttu-Bra clusters with members of the Brachyury subfamily in a phylogenetic analysis (Fig. 1B).

\subsection{Temporal expression pattern of Ttu-Bra}

The temporal expression profile of Ttu-Bra during Tubifex embryogenesis was analyzed by semi-quantitative RT-PCR on pooled embryos of defined stages. The results are shown in Fig. 2. The Ttu-Bra expression was first detected at stages 9-11, and abruptly down-regulated to almost undetectable level at stage 12 (see Fig. 3). The second round of Ttu-Bra expression took place at stages 16-17, although the amount of the transcripts was apparently much smaller than before (at stages 9-11).

\subsection{Spatial expression pattern of Ttu-Bra}

The first sign of Ttu-Bra expression was detected in a bilateral pair of micromeres in stage 10 embryos (i.e. shortly after division of 4D cell into a pair of endodermal precursors $\mathrm{E}^{\mathrm{D}}$ ). In earlier embryos (stages 1-8), there was no indication of Ttu-Bra expression (Fig. 4A 
and B). In embryos processed for in situ hybridization with a sense probe, no staining was detected from stage 1 to stage 18 (data not shown).

As Fig. $4 \mathrm{C}$ and D shows, the first Ttu-Bra-expressing cells were seen apposed to the dorsolateral margin of $\mathrm{M}$ teloblasts. Judging from their position in the embryo, the Ttu-Bra-expressing cells on the left and right side of the embryo correspond to micromeres 3d (Fig. 4C) and 3c (Fig. 4D), respectively. Ttu-Bra transcripts in these cells were still detectable at the beginning of stage 11 (Fig. 4E and F) but became undetectable within a few hours thereafter when a pair of endodermal precursors $\mathrm{E}^{\mathrm{D}}$ divided (see Fig. 5B).

Shortly after the cessation of Ttu-Bra expression in 3c and 3d, another set of micromeres located anteriorly began to express Ttu-Bra (Fig. 5C). These micromeres were located at the dorsal margin of endodermal precursors $\left(\mathrm{E}^{\mathrm{A}}\right.$ and $\left.\mathrm{E}^{\mathrm{B}}\right)$ and at the ventral margin of the micromere cap. More specifically, the righthand micromere was associated with $\mathrm{E}^{\mathrm{B}}$ (Fig. 5C) and the lefthand micromere with both $\mathrm{E}^{\mathrm{A}}$ and $\mathrm{E}^{\mathrm{B}}$ (Fig. 5B). Judging from their position, it is safe to say that these Ttu-Bra-expressing cells correspond to micromeres 3a and 3b. As in cells 3c and 3d, Ttu-Bra transcripts in these 'anterior' micromeres became undetectable within a short time; no signal was observed at the beginning of stage 12 when NOPQ divides into cells $\mathrm{N}$ and OPQ (data not shown).

Fig. 6A shows a schematic summary of the temporal patterns of Ttu-Bra expression in the third quartette of micromeres. In Tubifex, early cleavage cycles in the A/B cell lineages are significantly longer than those in the C/D cell lineages (Shimizu, 1995). As a result, cleavage asynchrony between these cell lineages becomes pronounced as development proceeds. At $22^{\circ} \mathrm{C}$ the division of cells $2 \mathrm{~A}$ and $2 \mathrm{~B}$ (into $3 \mathrm{a} / 3 \mathrm{~A}$ and $3 \mathrm{~b} / 3 \mathrm{~B}$, respectively) 
occurs 3.5 hrs later than the division of cells $2 \mathrm{C}$ and $2 \mathrm{D}$ into $3 \mathrm{c} / 3 \mathrm{C}$ and $3 \mathrm{~d} / 3 \mathrm{D}$, respectively (unpublished observation). That is to say, the birth of 3a and 3b lags 3.5 hrs behind the birth of 3c and 3d. The present results described above suggest that each of the third quartette of micromeres follows a similar time course of Ttu-Bra expression (Fig. 6A): Ttu-Bra expression begins at about 5 hrs after their birth; Ttu-Bra transcripts are detectable until the cells enter M phase, but become undetectable as M phase proceeds; and Ttu-Bra expression lasts for 2 hrs or so in all of the third micromeres. (In this study we could not determine the duration of Ttu-Bra expression because we do not know the precise time of Ttu-Bra expression cessation.) The initial Brachyury expression in the third micromeres (3c and 3d) has been reported in molluscs Patella vulgata (Lartillot et al., 2002) and Saccostrea kegaki (Kin et al., 2009), though some of the descendants of the second micromere 2d also express Brachyury in these organisms.

As to the above-mentioned spatial pattern of Ttu-Bra expression, it is summarized that the Ttu-Bra-expressing micromeres (3a-3d) comprise an arc that is running along the boundary between the anteriorly located cluster of micromeres (called the micromere cap) and yolky endodermal precursors derived from macromeres (Fig. 6B). It should be noted that this boundary corresponds topographically to the prospective stomodaeum (Anderson, 1973; Shimizu, 1982).

Consistent with the results of the RT-PCR analysis (Fig. 2), no Ttu-Bra-expressing cells were found in embryos at stages 12 to 15 . It was stage 16 when a second 'burst' of Ttu-Bra expression occurred in Tubifex embryos. As Fig. 7A-C shows, Ttu-Bra-expressing cells were found to be organized in a small ring at the posterior end of the embryo that 
corresponds to the prospective proctodaeum. As development proceeded to stage 17, the ring of Ttu-Bra-expressing cells appeared to reduce its diameter to form a cluster of cells (Fig. 7D-F). Similar spatial patterns of Brachyury expression have been reported in the caudal region of Drosophila embryos (Kispert et al., 1994).

In summary, Ttu-Bra is expressed twice during Tubifex embryogenesis, first in the third quartette of micromeres during early cleavage stages and then in the caudal region during final stages of body elongation; inbetween there is a rather long period ( 2 days) when no Ttu-Bra expression is detected (see Fig. 3O). As discussed earlier, the Ttu-Bra-expressing micromeres (3a-3d) are located in the region corresponding to the prospective stomodaeum (Fig. 6B); a caudal ring of Ttu-Bra-expressing cells in later embryos is located just at the prospective proctodaeum (Fig. 7D). This spatial pattern of Ttu-Bra expression apparently resembles the Brachyury expression patterns seen in the organisms that display an indirect mode of development via ciliary larvae, in that Brachyury is expressed in the two separate regions corresponding to the stomodaeum and the proctodauem (Tagawa et al., 1998; Shoguchi et al., 1999; Arendt et al., 2001; Croce et al., 2001; Lartillot et al., 2002; Kin et al., 2009).

If one considers the fact that like other clitellates, T. tubifex is a 'direct developer' that develops into a juvenile without any larval form, the stomodeal expression of Brachyury as seen in Tubifex could be regarded as an exceptional case, since the stomodeal Brachyury expression is undetectable in tunicates, acraniates, vertebrates, insects and nematodes, all of which develop directly or indirectly by means of larvae other than ciliary larvae (Yasuo and Satoh, 1994; Holland et al., 1995; Kispert et al., 1994; Woollard and Hodgkin, 2000). Only 
one exception is the direct developing chaetognath Paraspadella gotoi that exhibits Brachyury expression in two separate embryonic regions (Takada et al., 2002). Given that the original expression of Brachyury in Bilaterians was in the blastopore and its derived structures, the stomodaeum/foregut and the proctodaeum/hindgut in the primary larva (Technau, 2001) and that this original expression pattern might have been preserved in the polychaete annelid (Arendt et al., 2001), echinoderms (Shoguchi et al., 1999; Gross and McClay, 2001; Croce et al., 2001) and the hemichordate (Tagawa et al., 1998), it is likely that the anterior stomodeal expression of Brachyury (viz., one of the original traits of Brachyury expression) could have been preserved in the oligochaete lineage and the chaetognath lineage in spite of evolutionary transition to the direct mode of development.

\section{Experimental procedures}

\subsection{Embryos}

Embryos of freshwater oligochaete Tubifex tubifex were obtained as described previously (Shimizu, 1982) and cultured at $22^{\circ} \mathrm{C}$. For experiments, embryos were freed from cocoons in the culture medium (Shimizu, 1982). Unless otherwise stated, all experiments were carried out at room temperature $\left(20-22^{\circ} \mathrm{C}\right)$.

\subsection{RNA isolation and cDNA synthesis}

Total RNA was isolated from T. tubifex embryos by using ISOGEN (Nippon Gene) according to the manufacturer's nstructions. Poly (A) ${ }^{+}$RNA was isolated from total RNA using Oligotex-dT30<Super $>$ mRNA purification kit (TaKaRa) according to the supplier’s 
directions. First-strand cDNA for degenerate PCR and 3' rapid amplification of cDNA ends (3’RACE) was synthesized with a NotI-dT18 primer using PowerScript reverse transcriptase (Clonetech) according to the manufacturer's potocol. First-strand cDNA used as template for 5'RACE was synthesized with a gene-specific primer 3RACE-R1

(5’-CTCTGCCTGTGCGGATCG-3’) using a 5’RACE System for Rapid Amplification of cDNA Ends, version 2.0 (Life Technologies) according to the manufacturer's instructions.

\subsection{Degenerate PCR}

Degenerate primers specific to Brachyury genes were designed from amino acid sequences of T-box domain of the Brachyury-class genes from Platynereis dumerilii (annelid), Patella vulgata (mollusc), Drosophila melanogaster (fruit fly), Tribolium castaneum (beetle), Xenopus laevis (frog) and Mus musculus (mouse). A frward primer TbraF1 corresponded to amino acid sequence WKYVNG; a reverse primer TbraR1 corresponded to NPFAKA. Nucleotide sequences of these primers were 5’-TGGAA(A/G)TA(C/T)GT(A/G/C/T)AA(C/T)GG-3’(TbraF1) and 5'-GC(C/T)TT(A/G/C/T)GC(A/G)AA(A/G/C/T)GG(A/G)TT-3’'(TbraR1). Amplification parameters were: 10 cycles of $30 \mathrm{sec}$ at $94^{\circ} \mathrm{C}, 30 \mathrm{sec}$ at $60^{\circ} \mathrm{C}$ and $21 \mathrm{sec}$ at $72^{\circ} \mathrm{C}$ and 25 cycles of $30 \mathrm{sec}$ at $94^{\circ} \mathrm{C}, 30 \mathrm{sec}$ at $50^{\circ} \mathrm{C}$ and $21 \mathrm{sec}$ at $72^{\circ} \mathrm{C}$. The PCR product was gel-purified and directly cloned into pBluescriptII KS (+) vector. The inserted DNA fragments were sequenced using a BigDye Terminator v3.1 Cycle Sequencing kit (Applied Biosystems) with an ABI Prism 377 DNA Sequencer (Applied Biosystems). 


\subsection{Rapid amplification of cDNA ends (RACE)}

To isolate 3' and 5' portions of Ttu-Bra transcripts, 3' and 5'RACE were performed using gene-specific primers (GSPs) and adapter primers. Nucleotides sequences of the primers for 3'RACE were 5'-GTCGCAACATTCAGCTTCCC-3' (bra3RACE-F1), 5'-GTCGCTGTGACTGCCTACC-3'(bra3RACE-F2), and 5'-AACTGGAAGAATTCGCGGCC-3'(adapter primer). Nucleotide sequences of the primers for 5’RACE were 5'-GCGTGGCTGGTATTTGTGCAGTG-3’ (bra5RACE-F2), 5’-TCAACAGAATCTGTCCGTGGCCG-3’ (bra5RACE-F0), 5’-GGCCACGCGTCGACTAGTACGGGIIGGGIIGGGIIG-3’ (AP1), and 5’-GGCCACGCGTCGACTAGTAC-3’'(AP2).

3'RACE was performed, in two stages, using the following amplification parameters: 10 cycles (with bra3RACE-F1 primer only) of $30 \mathrm{sec}$ at $94^{\circ} \mathrm{C}, 30 \mathrm{sec}$ at $57^{\circ} \mathrm{C}$ and $2 \mathrm{~min}$ at $72^{\circ} \mathrm{C}$ and additional 20 cycles (with bra3RACE-F1 and adaptor primer) of 30 sec at $94^{\circ} \mathrm{C}, 30$ sec at $60^{\circ} \mathrm{C}$ and $2 \mathrm{~min}$ at $72^{\circ} \mathrm{C}$. Next, nested PCR was carried out in the same way as the first PCR except for using bra3RACE-F2 as a GSP.

5'RACE was performed using the following amplification parameters: 40 cycles (with bra5RACE-F2 and AP1) of $1 \mathrm{~min}$ at $94^{\circ} \mathrm{C}, 30 \mathrm{sec}$ at $55^{\circ} \mathrm{C}$ and $2 \mathrm{~min}$ at $72^{\circ} \mathrm{C}$. This PCR product was used as a template for nested PCR with bra5RACE-F0 and AP2; amplification parameters were: 35 cycles of $30 \mathrm{sec}$ at $94^{\circ} \mathrm{C}, 30 \mathrm{sec}$ at $65^{\circ} \mathrm{C}$ and $1 \mathrm{~min}$ at $72^{\circ} \mathrm{C}$. PCR products were gel purified, cloned into pBluescriptII KS (+), and sequenced as described above. 


\subsection{Semi-quantitative developmental RT-PCR}

Templates for semi-quantitative RT-PCR were synthesized from total RNAs extracted from several hundred embryos at each stage with a NotI-dT18 primer using PowerScript reverse transcriptase (Clonetech) according to the supplier’s instructions. Ttu-Bra fragments (nt 703 - 1783) were amplified using GSPs (bra3RACE-F1 and bra3RACE-R1). Amplification parameters were: 35 cycles of $30 \mathrm{sec}$ at $94^{\circ} \mathrm{C}, 30 \mathrm{sec}$ at $55^{\circ} \mathrm{C}$ and $40 \mathrm{sec}$ at $72^{\circ} \mathrm{C}$. As an internal standard to adjust for differences in efficiencies of RNA extraction and first strand cDNA synthesis between each sample, 450-bp long fragment for Tubifex $\beta$-actin was amplified in parallel to each sample. Amplification parameters were: 30 cycles of $30 \mathrm{sec}$ at $94^{\circ} \mathrm{C}, 30 \mathrm{sec}$ at $52^{\circ} \mathrm{C}$ and $30 \mathrm{sec}$ at $72^{\circ} \mathrm{C}$. To quantitate PCR products, each sample was electrophoresed in 1.5\% agarose gel and stained with ethidium bromide. Photograph for the UV-transilluminated gel was scanned and band intensity was measured using NIH image analyzer software.

\subsection{Whole-mount in situ hybridization}

DIG-labeled RNA probes were prepared according to the protocols described by Matsuo et al. (2005). Templates for riboprobe synthesis were amplified, with an M13 primer set, from the plasmids containing cDNA fragments of Ttu-Bra (nt 829-1498 bp). An antisense and sense riboprobes were synthesized with T7 and T3 RNA polymerases, respectively. Whole-mount in situ hybridization was performed according to the method described previously (Oyama and Shimizu, 2007) with slight modifications. Hybridization (60-72 h) and subsequent wash were both performed at $55^{\circ} \mathrm{C}$. 
Whole-mount processed (stained) embryos were fixed with 3.5\% formaldehyde in phosphate buffer ( $\mathrm{pH}$ 7.4) for $12 \mathrm{hr}$, mounted in PBS (+ 0.1\% Tween-20), and observed under incident light.

\section{Acknowledgements}

We thank members of the Shimizu laboratory for advice and help in collecting embryos. This study was supported in part by a Grant-in-Aid from the Ministry of Education, Science, Sports and Culture, Japan (13680799) to T.S.

\section{References}

Anderson, D. T., 1973. Embryology and Phylogeny in Annelids and Arthropods. Pergamon, Oxford.

Arendt, D., Technau, U., Wittbrodt, J., 2001. Evolution of the bilaterian larval foregut. Nature 409, 81-85.

Croce, J., Lhomond, G., Christian, G., 2001. Expression pattern of Brachyury in the embryo of the sea urchin Paracentrotus lividus. Dev. Genes Evol. 211, 617-619.

Gross, J. M., McClay, D. R., 2001. The role of Brachyury (T) during gastrulation movements in the sea urchin Lytechinus variegatus. Dev. Biol. 239, 132-147.

Harada, Y., Yasuo, H., Satoh, N., 1995. A sea urchin homologue of the chordate Brachyury (T) gene is expressed in the secondary mesenchyme founder cells. Development $121,2747-2754$.

Hejnol, A., Martindale, M. Q., 2008. Acoel development indicates the independent 
evolution of the bilaterian mouth and anus. Nature 456, 382-386.

Holland, P. W. H., Koschorz, B., Holland, L. Z., Herrmann, B. G., $1995 . \quad$ Conservation of Brachyury (T) genes in amphioxus and vertebrates: developmental and evolutionary implications. Development 121, 4283-4291.

Kin, K., Kakoi, S., Wada, H., 2009. A novel role for dpp in the shaping of bivalve shells revealed in a conserved molluscan developmental program. Dev. Biol. 329, 152-166.

Kispert A, Herrmann BG, Leptin M and Reuter R (1994) Homologs of the mouse Brachyury gene are involved in the specification of posterior terminal structures in Drosophila, Tribolium, and Locusta Genes Dev. 8: 2137-2150.

Kusch, T., Reuter, R., 1999. Functions fr Drosophila brachyenteron and forkhead in mesoderm specification and cell signaling. Development 126, 3991-4003.

Lartillot, N., Lespinet, O., Vervoort, M., Adoutte, A., 2002. Expression pattern of Brachyury in the mollusc Patella vulgata suggests a conserved role in the establishment of the AP axis in Bilateria. Development 129, 1411-1421.

Matsuo, K., Yoshida, H., Shimizu, T., 2005. Differential expression of caudal and dorsal genes in the teloblast lineages of the oligochaete annelid Tubifex tubifex. Dev. Genes Evol. 215, 238-247.

Oyama, A., Shimizu, T., 2007. Transient occurrence of vasa-expressing cells in nongenital segments during embryonic development in the oligochaete annelid Tubifex tubifex. Dev. Genes Evol. 217, 675-690.

Scholz, C. B., Technau, U., 2001. The ancestral role of Brachyury: expression of NemBra1 
in the basal cnidarian Nematostella vectensis (Anthozoa). Dev. Genes Evol. 212, 563-570.

Shimizu, T., 1982. Development in the freshwater oligochaete Tubifex. In Harrison, F. W., Cowden, R., R. (Eds), Developmental Biology of Freshwater Invertebrates. Alan R. Liss, New York, 283-316.

Shimizu, T., 1995. Lineage-specific alteration in cell cycle structure in early Tubifex embryos. Dev. Growth Differ. 37, 263-272.

Shimizu, T., Nakamoto, A., 2001. Segmentation in annelids: cellular and molecular basis for metameric body plan. Zool. Sci. 18, 285-298.

Shoguchi, E., Satoh, N., Maruyama, Y. K., 1999. Pattern of Brachyury gene expression in starfish embryos resembles that of hemichordate embryos but not of sea urchin embryos. Mech. Dev. 82, 185-189.

Showell, C., Binder, O., Conlon, F. L., 2004. T-box genes in early embryogenesis. $\quad$ Dev. Dyn. 229, 201-218.

Tagawa, K., Humphrey, T., Satoh, N., 1998. Novel pattern of Brachyury gene expression in hemichordate embryos. Mech. Dev. 75, 139-143.

Takada, N., Goto, T., Satoh, N., 2002. Expression pattern of the Brachyury gene in the arrow worm Paraspadella gotoi (Chaetognatha). Genesis 32, 240-245.

Technau, U., 2001. Brachyury, the blastopore and the evolution of the mesoderm. BioEssays 23, 788-794.

Yasuo, H., Satoh, N., 1994. An ascidian homologue of the mouse Brachyury (T) gene is expressed exclusively in notochord cells at the fate restricted stage. Dev. Growth 
Differ. 36, 9-18.

Woollard, A., Hodgkin, J., 2000. The Caenorhabditis elegans fate-determining gene mab-9 encodes a T-box protein required to pattern the posterior hindgut. Genes Dev. 14, 596-603. 


\section{Figure legends}

Fig. 1. Characterization of Ttu-Bra, a Brachyury homologue from Tubifex tubifex. (A) Alignment of the T-domain of Ttu-Bra with known Brachyury class proteins. The numbers of amino acid residues in the T-domain presented are 222 for Ttu-Bra, 218 for Pdu-Bra and Pvu-Bra, and 216 for Xla-Bra, Mmu-T and Dme-Byn. Asterisks represent amino acid identity. Numbers in parentheses indicate the percentage amino acid identity with the T-domain of Ttu-Bra. $\quad$ (B) Molecular phylogenetic relationship of Ttu-Bra to other T-box proteins. The phylogenetic tree was generated by the neighbor joining method using PAUP*4.0b10. Mmu-Tbr-1 and Xla-Eomes were used as outgroups. Numbers ae bootstrap values (as percentages of 1000 replications). Lengths of branches are drawn to the scale indicated. Species abbreviations: Bfl, Branchiostoma floridae (amphioxus); Cel, Caenorhabditis elegans (nematode); Dme Drosophila melanogaster (fruit fly); Gga, Gallus gallus (chick); Hpu, Hemicentrotus pulcherrimus (sea urchin); Hro, Halocynthia rotetzi (tunicate); Hsa, Homo sapiens (human); Hvu, Hydra vulgaris (hydra); Mmu Mus musculus (mouse); Nve, Nematostella vectensis (cnidaria); Pdu, Platynereis dumerilli (annelid); Pvu, Patella vulgata (mollusc); Tca, Tribolium castaneum (beetle); Ttu, Tubifex tubifex (annelid); Xla, Xenopus laevis (frog).

Fig. 2. Temporal expression profile of Ttu-Bra. Semi-quantitative RT-PCR analysis showing the relative levels of Ttu-Bra transcripts in T. tubifex embryos from stage 1 to stage 18, with stages 2-4 defined as 1. $\quad \beta$-Actin was used as internal control. Ttu-Bra transcripts were detected at stage 9-11 and stage 16-17. 
Fig. 3. Summary of Tubifex development. Diagrammatic illustration of selected stages of embryonic development. (A-E) Animal pole views of embryos at stages 1-cell (A), 2-cell (B), 4-cell (C), 8-cell (D) and 10-cell (E). (F) Stage 8 embryo. Posterior view with dorsal to the top. Three cells $\left(2 \mathrm{~d}^{11}\right.$, $4 \mathrm{~d}$ and $\left.4 \mathrm{D}\right)$ of the $\mathrm{D}$ cell line come to lie in the future midline. (G) Stage 11 embryo with a bilateral pair of ectoteloblast precursors (NOPQl, NOPQr), mesoteloblasts $(\mathrm{Ml}, \mathrm{Mr})$ and D-cell line endodermal precursors $\left(\mathrm{E}^{\mathrm{D}}\right) . \quad(\mathrm{H})$ Stage 12c embryo at the completion of teloblastogenesis. Dorsal view with anterior to the pp. (I-N) Left side views of embryos (with anterior to the left) undergoing gastrulation (stages 13-15) and body elongation (stages 16-18). Gastrulation in Tubifex consists of ventralward movement of elongating germ bands (shaded) and spreading of micromere-derived epithelial cells (not depicted here) over the endoderm. Body elongation is accompanied by formation of segmental ectoderm, which is accomplished by dorsalward expansion of germ bands (shaded in L-N). Asterisks indicate the position of buccal cavity. mc, micromere cap; pr, prostomium. (For details of morphogenetic movements, see Shimizu and Nakamoto, 2001) (O) Time course of Tubifex development (stages 1 to 18 at $22^{\circ} \mathrm{C}$ ).

Fig. 4. Expression patterns of Ttu-Bra. (A and B) Left-side and right-side views of a stage 8 embryo. Dorsal is to the top. Cells 2d, 4d and 4D are indicated. Note that there are no Ttu-Bra-expressing cells. The inset shows a diagram of stage 8 embryo (posterior view) with viewing directions (arrows A and B) for panels A and B. (C and D) Left-side and right-side views of a stage 10 embryo. Dorsal is to the top. Note the localization of 
Ttu-Bra transcripts in two micromeres (arrowheads). M teloblasts and endodermal precursors $\mathrm{E}^{\mathrm{C}}$ and $\mathrm{E}^{\mathrm{D}}$ (derived from C-cell and D-cell line macromeres) are indicated. The inset shows a diagram of stage 10 embryo (posterior view) with viewing directions (arrows C and D) for panels C and D. (E and F) Left-side and right-side views of an early stage 11 embryo (shortly after division of 2d into a pair of NOPQ). Arrowheads indicate Ttu-Bra-expressing cells. The inset shows a diagram of stage 11 embryo (posterior view) with viewing directions (arrows E and F) for panels E and F. Scale bar in (A): (A-F) 200 $\mu \mathrm{m}$.

Fig. 5. Expression of Ttu-Bra in mid stage 11. A mid stage 11 embryo is viewed posteriorly (A), anterolaterally (B) and anteriorly (C). Dorsal is to the top. The inset shows a diagram of stage 11 embryo (dorsal view with posterior to the top) with viewing directions (arrows A, B and C) for panels A, B and C. Ttu-Bra-expressing cells (arrowheads) are associated not only with the dorsoanterior margin of endodermal precursors $\mathrm{E}^{\mathrm{A}}$ and/or $\mathrm{E}^{\mathrm{B}}$ (derived from macromeres $3 \mathrm{~A}$ and $3 \mathrm{~B}$ ) but also with the ventral margin of the micromere cap (mc). The asterisk in (B) indicates the approximate position of previous micromere 3d. Scale bar in (A): (A-C) $200 \mu \mathrm{m}$.

Fig. 6. (A) Time course of Ttu-Bra expression in the third micromeres (3a-3d) at $22^{\circ} \mathrm{C}$. For brevity, cells 3b and 3c are omitted. The period when Ttu-Bra expression is detected is indicated by horizontal, thick bars as a function of time (hrs) after division of the 2D cell into 3d and 3D. 3a (3b) is formed at about 3.5 hrs after the 3d (3c) birth. Vertical arrows 
indicate the approximate time of cell division. (B) Diagrammatic illustration of stage 8 embryo viewed from left anterior (with dorsal to the top) and dorsal (with anterior to the left). Note the embryonic position of the third micromeres (colored in red) relative to other micromeres and macromeres (endodermal precursors).

Fig. 7. Expression patterns of Ttu-Bra. (A-C) A stage 16 embryo viewed from the left-side (A) and from the dorsal side (B and C). Anterior is to the left. Ttu-Bra-expressing cells are localized to the caudal end of the embryo and are organized in a circle (arrowheads). (D-F) A stage 17 embryo viewed from the right-side (D) and from the dorsal side (E and F). Posterior is to the top. Arrowheads indicate a cluster of Ttu-Bra-expressing cells located at the caudal end of the embryo. Scale bar in (A): (A, B, D, E) $200 \mu \mathrm{m}$; (C, F) $100 \mu \mathrm{m}$. 
Ttu-Bra

Pdu-Bra

Pvu-Bra

Xla-Bra

Mmu-T

Dme-Byn

Ttu-Bra

Pdu-Bra

Pvu-Bra

Xla-Bra

Mmu-T

Dme-Byn

Ttu-Bra

Pdu-Bra

Pvu-Bra

Xla-Bra

Mmu-T

Dme-Byn
LDDRQLWAQFQQLTNEMIVTKSGRRMFPVIRVSVSGLDASAMYSMLLDFVPADEHRWKYVNGEWSPCGKSQHQAT LEDRELWGKFKELTNEMIVTKSGRRMFPVLRASVTGLDPNAMYSFLLDLVNVDNHRWKYVNGDWVPGGKAEPQPH LEDRSLWEKFKEFTNEMIVTKNGRRMFPVFRVSVSGVDPNAMYTLLLDFVQVDNHRWKYVNGDWVPGGKAEPAAP LEERDLWTRFKELTNEMIVTKNGRRMFPVLKVSMSGLDPNAMYTVLLDFVAADNHRWKYVNGEWVPGGKPEPQAP LEESELWLRFKELTNEMIVTKNGRRMFPVLKVNVSGLDPNAMYSFLLDFVTADNHRWKYVNGEWVPGGKPEPQAP LDDRELWLRFQNLTNEMIVTKNGRRMFPVVKISASGLDPAAMYTVLLEFVQIDSHRWKYVNGEWVPGGKAEVPPS

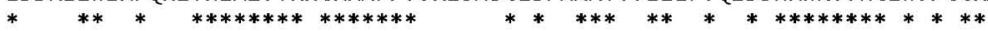

PTPSSGPSNVYVHPDSPNFGAHWMKEPVSFGKVKLSNKQHGHGQILLNSLHKYQPRIHIARVAE---NNNVATFS -----NAVYIHPDSPNFGAHWMKEPVSFSKVKLTNKMHGSGQIMLNSLHKYQPRIHIVKVGNKDEKRTISTHD

-----NCVYIHPDSPNFGAHWMKEPLSFSKVKHTNKLNGGGQIML NSLHKYGPRIHIVRVNSRTQKKSIMTFS

-----SCVYIHPDSPNFGAHWMKDPVSFSKVKL TNKMNGGGQIMLNSLHKYEPRIHIVRVGG--TQRMITSHS -SCVYIHPDSPNFGAHWMKAPVSFSKVKLTNKLNGGGQIMLNSLHKYEPRIHIVRVGG --PQRMITSHC

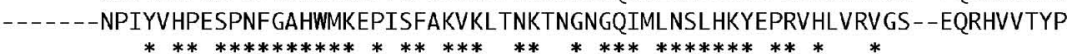

FPETTFVAVTAYQNEEITSLKIKYNPFAKAFQDT (100\%)

FVETQFVAVTAYQNEEITALKIKYNPFAKAFQDA ( $72 \%$ )

FPETQFIAVTAYQNEEITSLKIKHNPFAKAFLDA ( $70 \%)$

FPETQFIAVTAYQNEEITALKIKHNPFAKAFLDA ( $72 \%$ )

FPETQFIAVTAYQNEEITALKIKYNPFAKAFLDA ( $72 \%$ )

FPETQFIAVTAYQNEEVTSLKIKYNPFAKAFLDA ( $69 \%)$

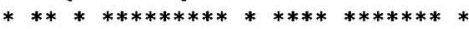

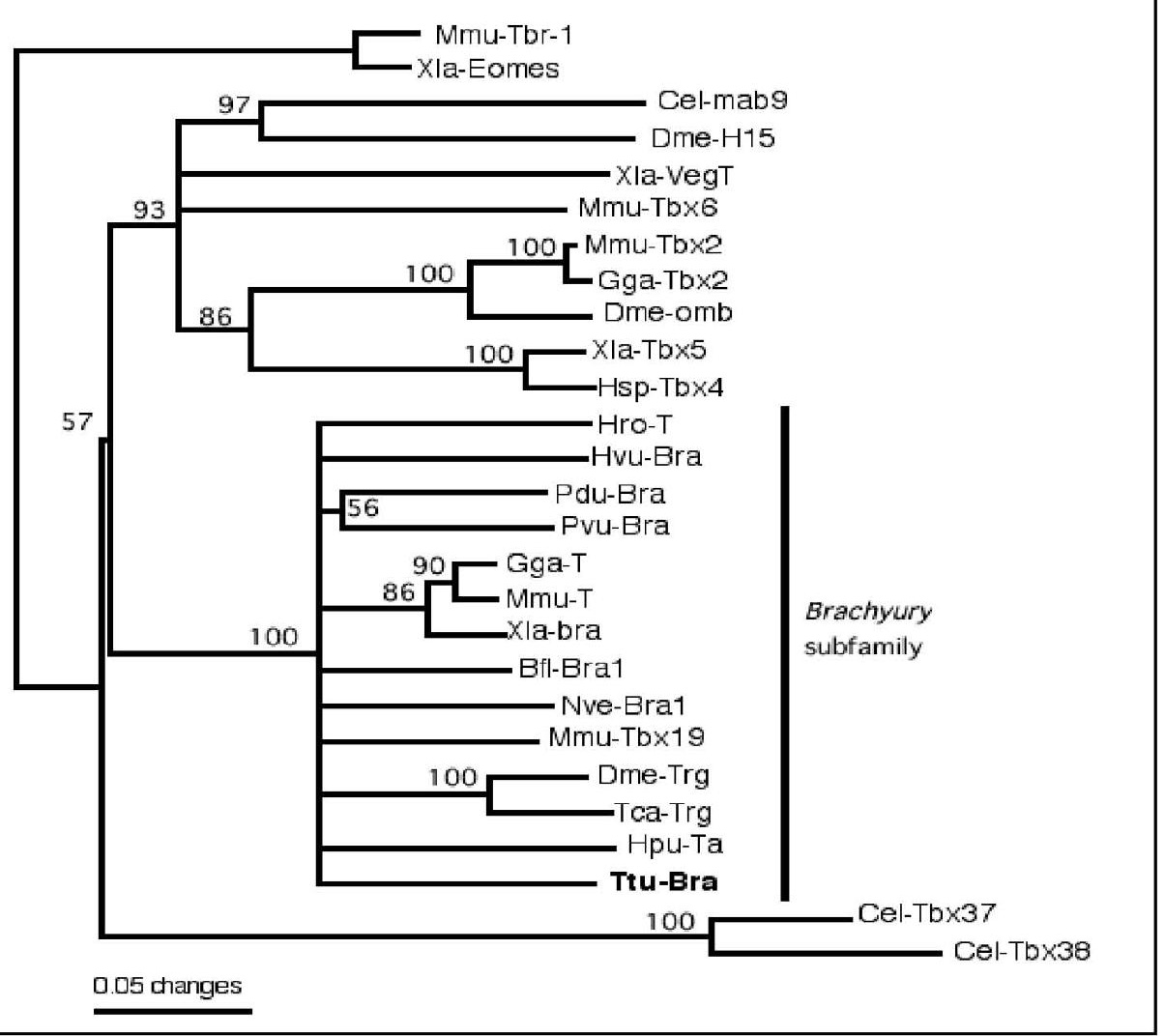




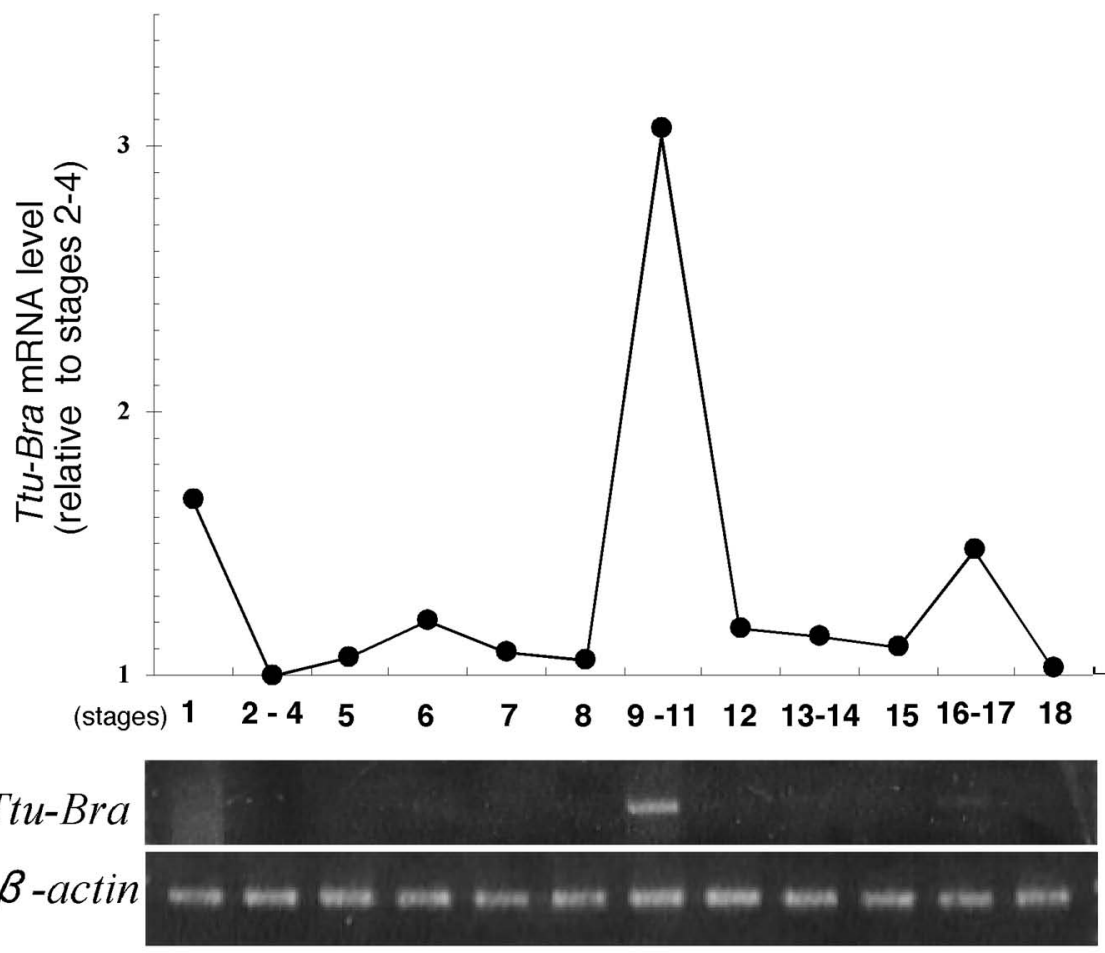



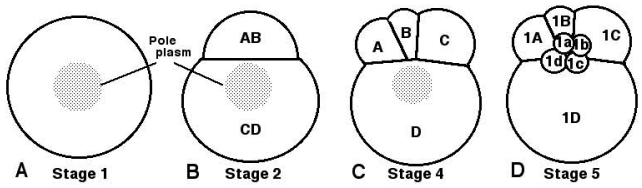

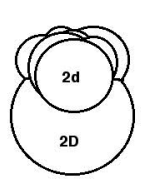

E Stage 6
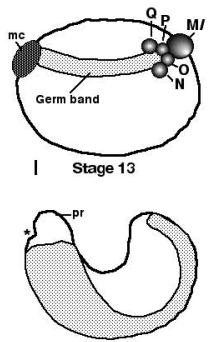

L Stage 16

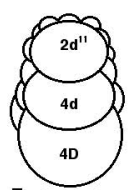

F stage 8

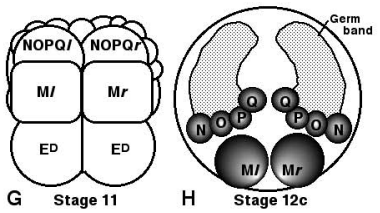

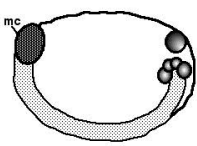

J Stage 14

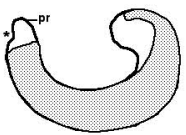

M Stage 17
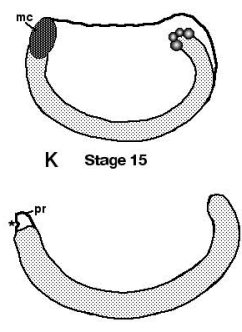

N Stage 18

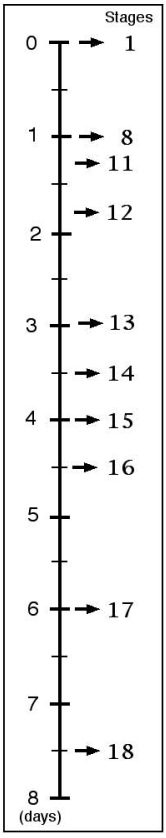




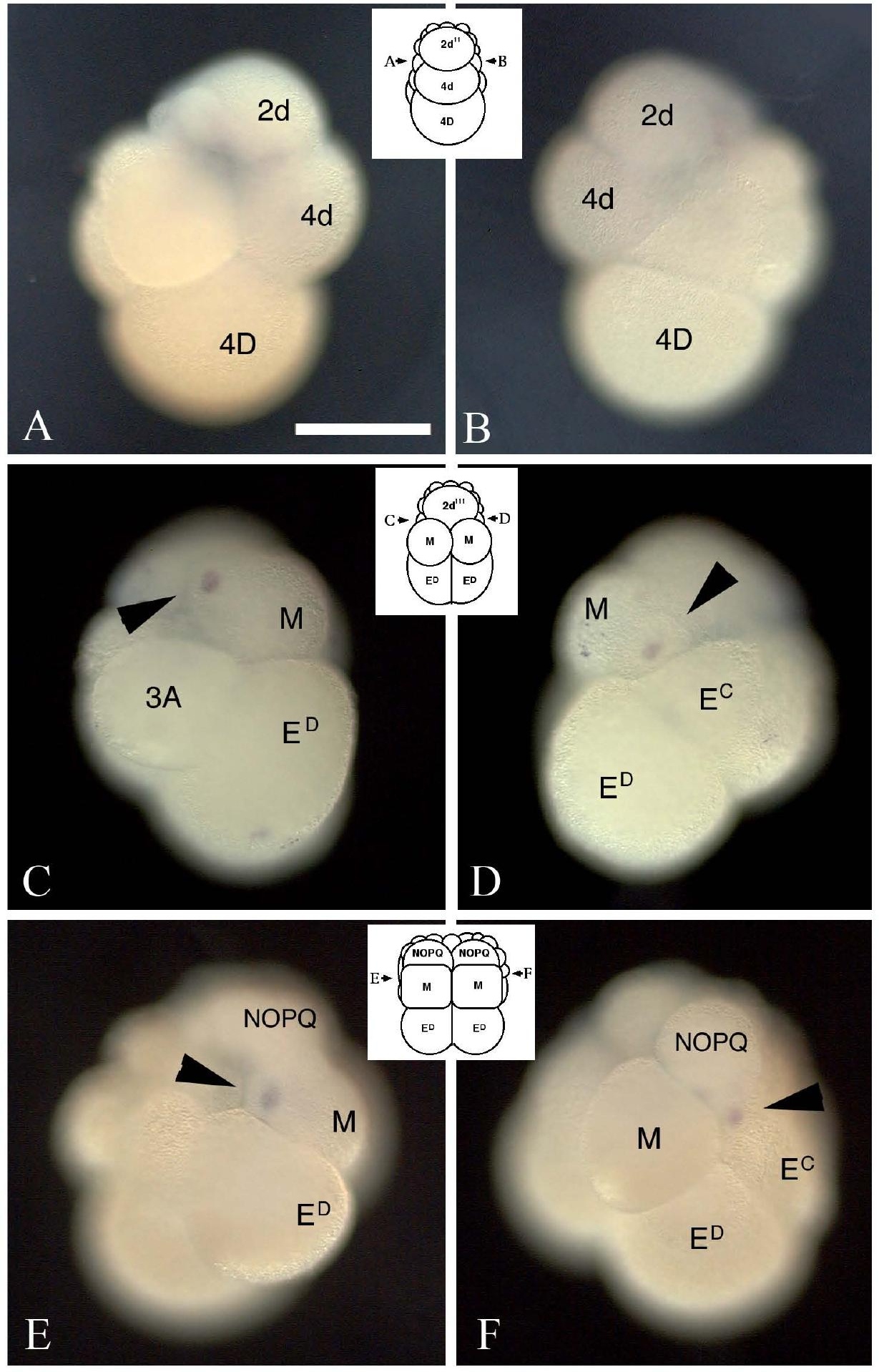




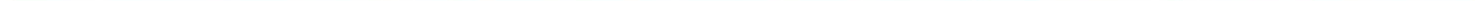




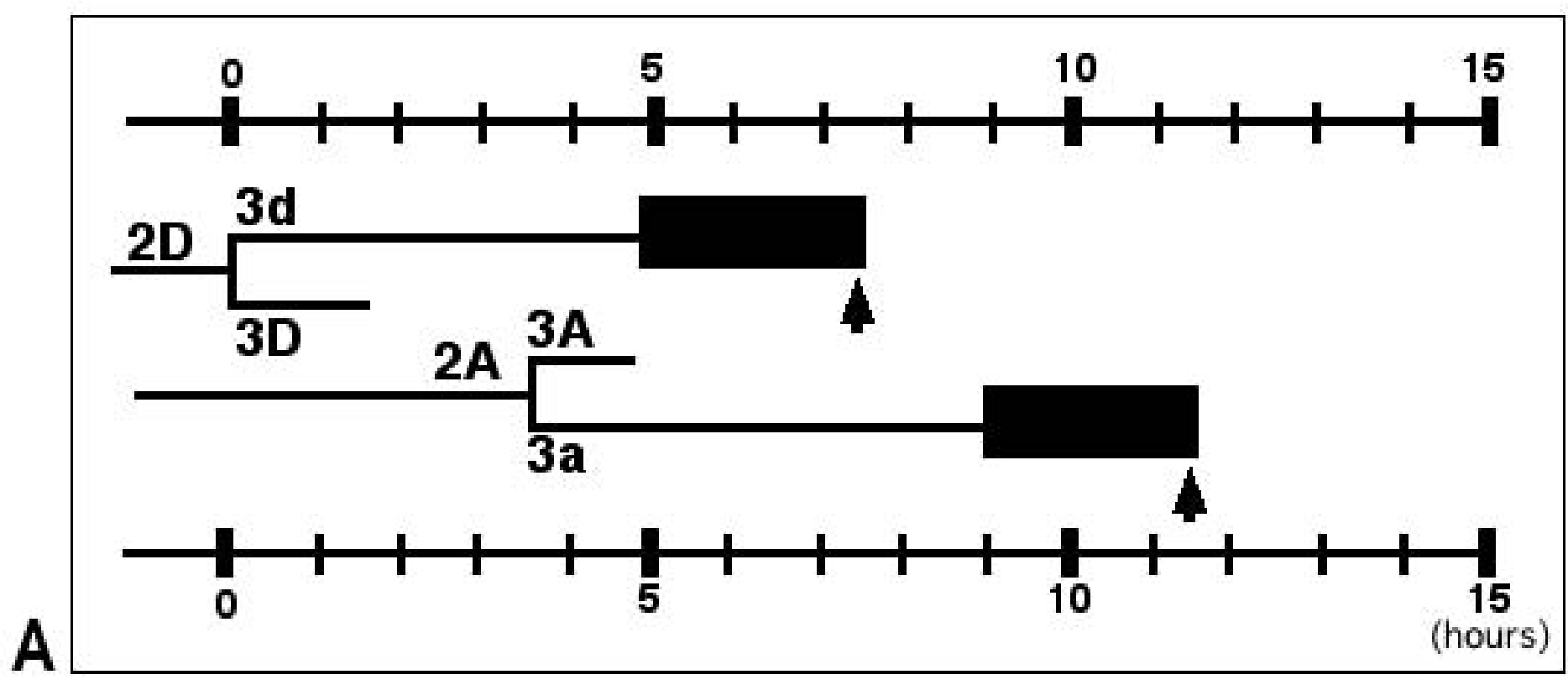

B

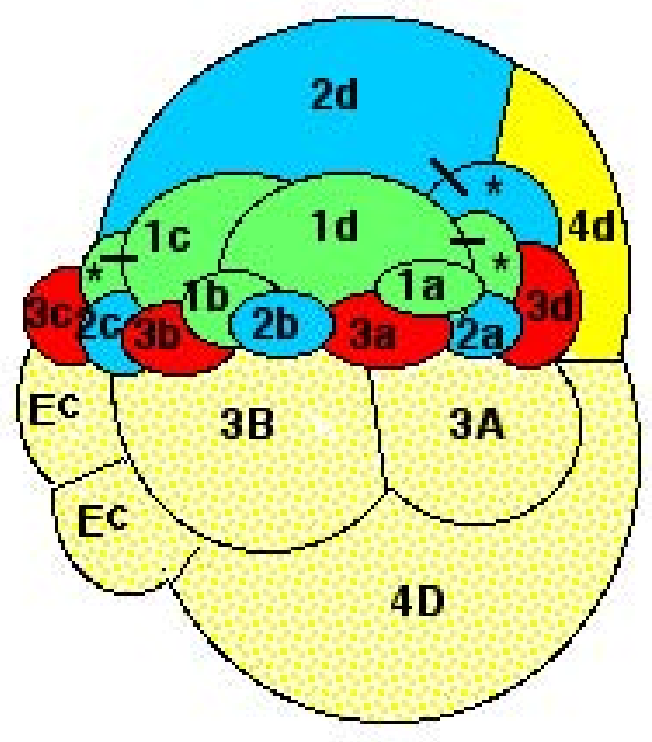

Left anterior view

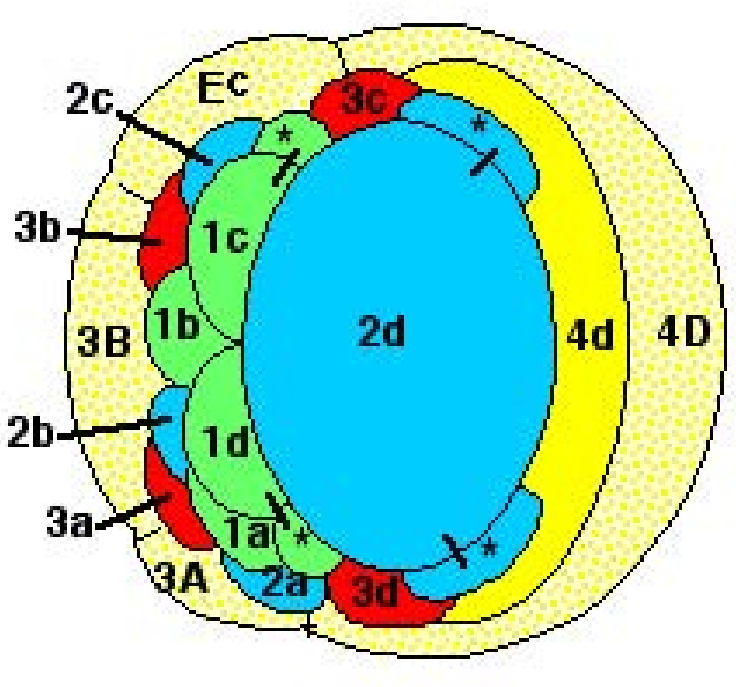

Dorsal view 


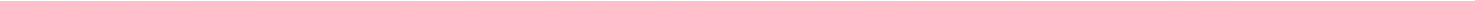

